To the Editors:

\title{
Dengue haemorrhagic fever in a child presenting as encephalitis on day one of fever
}

\author{
Ceylon Medical Journal 2012; 57: 177
}

The leading article on pathogenesis of severe dengue infection in your previous issue was very informative and covers a wide range of manifestations of dengue [1]. We would like to share our experience of a child with dengue haemorrhagic fever presenting as encephalitis on day one of fever.

A nine-year old boy presented on first day of fever with behavioural change, headache and vomiting. He was immunised against Japanese encephalitis. Examination revealed an obese, febrile, drowsy and disoriented child with neck stiffness. His eye opening was spontaneous, spoke inappropriate words and localised the pain. Glasgow coma scale was 12. Cardiovascular, respiratory and abdominal examinations were normal. His blood sugar and serum electrolytes were normal. The white cell count was $16.1 \times 10^{6} / \mu \mathrm{l}(\mathrm{N}-71 \%, \mathrm{~L}-22 \%)$. The haemoglobin was $13 \mathrm{~g} / \mathrm{dl}$, haematocrit was $39.7 \%$ and platelet count was $332 \times 10 \% / \mu 1$.

A clinical diagnosis of meningoencephalitis was made. Intravenous (IV) dexamethasone and IV cefotaxime were started. On the second day, child was having hallucinations. A non-contrast CT scan of head showed cerebral oedema. Electroencephalogram showed diffuse background slowing in the range of delta/theta compatible with encephalitis. intravenous mannitol and IV acyclovir were started. He showed clinical improvement by the fifth day but fever spikes continued. Abdommal ultrasonography showed a thickened gall bladder wall with pericholecystic fluid, mild ascites and hepatomegaly. There was thrombocytopenia $\left(15 \times 10^{6} / \mu \mathrm{l}\right)$ leucopenia $\left(3.4 \times 10^{6} /\right.$ $\mu \mathrm{l})$ and a rise in haematocrit (43.8\%). A diagnosis of dengue haemorrhagic fever (DHF) was made and managed accordingly. Cerebrospinal fluid (CSF) analysis did not show a cellular response. Dengue IgM and IgG were detected in both CSF and blood using immunochromatographic rapid detection test (Hexagon Dengue). The child made a complete recovery.

Neurologic manifestations including transverse myelitis, Guillain-Barré syndrome, acute disseminated encephalomyelitis and acute motor weakness have been reported in dengue patients [2,3]. Dengue encephalitis is defined as fever, headache, reduced consciousness not explained by liver failure, electrolyte derangement, shock, intracranial haemorrhage, dengue IgM in CSF or serum and neuroimaging suggestive of viral encephalitis [2]. Encephalitis on the first day of fever is a rare manifestation of dengue.

\section{References}

1. Malavige G N, Ogg G. Pathogenesis of severe dengue infection. The Ceylon Medical Journal 2012; 57: 97-100.

2. Varatharaj A. Encephalitis in the clinical spectrum of dengue infection. Neurology India 2010; 58: 585-91.

3. Misra UK, Kalita J, Syam UK, Dhole TN. Neurological manifestations of dengue virus infection. Journal of the Neurological Sciences 2006; 244: 117-22.

\footnotetext{
S Abeywardana ${ }^{1}$, PJ P Peiris ${ }^{1}$, A C M Gunasekara', V P Sinhabahu ${ }^{1}$

${ }^{1}$ Paediatric and ${ }^{2}$ Neurology Units, Provincial General Hospital, Ratnapura, Sri Lanka.

Correspondence: VPS, e-mail: <sinbad.lk@gmail.com >. Received 12 September and revised version accepted 22 October 2012. Competing interests: none declared.
} 